\title{
CO-OCCURRENCE VECTORS FROM CORPORA VS. DIS'TANCE VECTORS FROM DICTIONARIES
}

\author{
Yoshiki Niwa and Yoshihiko Nitta \\ Advanced Research Laboratory, Mitachi, Ltd. \\ Hatoyama, Saitama 350-03, Japan \\ \{niwa2, nitta\}@harl.hitachi.co.jp
}

\begin{abstract}
A comparison was made of vectors derived by using ordinary co-occurrence statistics from large text corpora and of vectors derived by measuring the interword distances in dictionary definitions. The precision of word sense disambiguation by using co-occurrence vectors from the 1987 Wall Street Journal (20M total words) was higher than that by using distance vectors from the Collins Finglish Dictionary (60K head words $+1.6 \mathrm{M}$ definition words). Ilowever, other experimental results suggest that distance vectors contain some different semantic information from co-occurrence vectors.
\end{abstract}

\section{Introduction}

Word vectors reflecting word meanings are expected to enable numerical approaches to semantics. Some early attempts at vector representation in psycholinguistics were the semantic differential approach (Osgood et al. 1957) and the associalive distribution approach (Deese 1962). However, they were derived mamally through psychological experiments. An early attempt at automation was made by Wilks et al. (1990) ulsing co-occurrence statistics. Since then, there lave been some promising results from using co-occurrence vectors, such as word sense disambigutition (Scluitte. 1993), and word clustering (Pereira ct al. 1993).

llowever, using the co-occurrence statislics requires a huge corpus that covers even most rare words. We recently developed word vectors that are derived from an ordinary dictionary by measuring the interword distances in the word definilions (Niwa and Nitta 1993). 'This method, by its nature, has no problem handling rare words. In this paper we examine the usefulness of these distance vectors as semantic representations by comparing them with co-occurrence vectors.

\section{Distance Vectors}

A reference network of the words in a dictionary (Fig. 1) is used to measure the distance between words. 'The network is a graph that shows which words are used in the definition of eacl, word (Nitta 1988). 'The network shown in lig. 1 is for a very small portion of the reference network for the Collins Ruglish Dictionary (1979 edition) in the CD-ROM I (Liberman 1991), with 60K head words $+1.6 \mathrm{M}$ definition words.

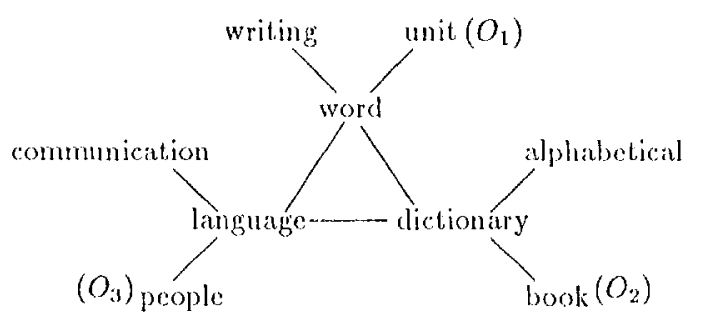

Fig. 1 Portion of a reference network.

For example, the definition for diclionary is $" \mathrm{a}$ book in which the words of a language are listed alphabetically ... " The word dictionary is thus linked to the words book, mord, language, and alphabetical.

A word vector is defined as the list of distances from a word to a certain set of selected words, which we call origins. The words in Fig. 1 marked with $O_{i}$ (unil, book, and people) are assumed to be origin words. In principle, origin words can be freely chosen In our experiments we used middle frequency words: t.ler 51st to 1050th most frequent words in the refercure Collins binglisla Dichionary (CHD)).

The distance vector for dictionary is derived as follows:

$$
\text { diclionary } \Rightarrow\left(\begin{array}{c}
2 \\
1 \\
2
\end{array}\right) \begin{aligned}
& \cdots \text { distance }\left(\text { dict. }, O_{1}\right) \\
& \cdots \text { distance }\left(\text { dict., } O_{2}\right) \\
& \cdots \text { distance }\left(\text { dict. }, O_{3}\right)
\end{aligned}
$$

The $i$ th element is; the distance (the length of the shortest path) between dictionary and the i-th origin, $O_{i}$. To begint, we assume every link has a constant length of 1. The actual clefinition for link length will be given later.

If word $A$ is used in the definition of word $R$, these words are expected to be strongly related. 'This is the basis of our hypothesis that the distances in the reference network reflect the associative distances between words (Nittia 1993). 
Use of Reference Networks Reference networks have been successfully used as neural networks (by Véronis and Ide (1990) for word sense disarrbiguation) and as fields for artificial association, such as spreading activation (by Kojima and Furugori (1993) for context-coherence measurement). 'The distance vector of a word can be considered to be a list of the activation strengths at the origin nodes when the word node is activated. 'Therefore, clistance vectors can be expected to convey almost the same information as the entire network, and clearly they are much easier to handle.

Dependence on Dictionaries $\Lambda$ s a semantic representation of words, distance vectors are expected to depend very weakly on the particular source dictionary. We compared two sets of distance vectors, one from LIDOCE (Procter 1978) and the other from COBUIIJ) (Sinclair 1987), and verified that their ditference is at least smaller than the diference of the word definitions themselves (Niwa and Nitta 199:3).

We will now describe some technical details ahout the derivation of distance vectors.

Link Length Distance measurement in a reference network depends on the definition of link lengtls. Previously, we assumed for simplicity that every link has a constant length. Mowever, this simple definition seems unnatural because it does not reflect word frequency. Because a path through low-frequency worels (rare words) implies a strong relation, it shonld be measured as a shorter path. Therefore, we use the following definition of link length, which takes account of word frequency.

$$
\text { length }\left(W_{1}, W_{2}\right) \underset{\text { def }}{=}-\log \left(\frac{n^{2}}{N_{1} \cdot N_{2}}\right)
$$

This shows the length of the links between words $W_{i}(i=1,2)$ in Fig. 2, where $N_{i}$ denotes the total number of links from and to $W_{i}$ and $n$ denotes the mumber of direct links between these: two words.

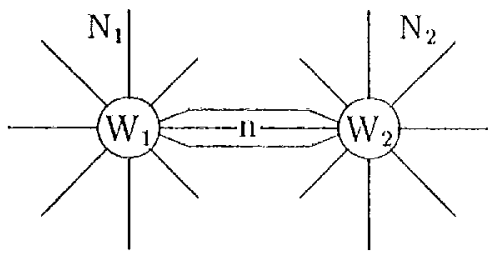

Fig. 2 Links between two words.

Normalization Distance vectors are normalized by first changing each coorclinate into its cleviation in the coordinate:

$$
v=\left(v_{i}\right) \quad \ldots \quad v^{\prime}=\left(\frac{v_{i}-a_{i}}{\sigma_{i}}\right),
$$

where $a_{i}$ and $\sigma_{i}$ are the average and the standard cleviation of the distances from the $i$-th origin. Next, each coordinate is changed into its deviation in the vector:

$$
v^{\prime}=\left(v_{i}^{\prime}\right) \quad \rightarrow \quad \vec{v}=\left(\frac{v_{i}^{\prime}-\overrightarrow{v^{\prime}}}{\sigma^{\prime}}\right)
$$

where $\overrightarrow{\eta^{\prime}}$ and $\sigma^{\prime}$ are the average and the standard devialion of $v_{i}^{\prime}(i=1, \ldots)$.

\section{Co-occurrence Vectors}

We use ordinary co-occurrence statisties and measure the co-occurrence likelihood between two words, $X$ and $Y$, by the mutual information estimate (Church and Ilanks 1989):

$$
I(X, Y)=\log ^{+} \frac{P(X \mid Y)}{P^{\prime}(X)}
$$

where $P(X)$ is the occurrence density of word $X$ in a whole corpus, and the conditional probability $\mathrm{P}^{2}(\mathrm{X} \mid \mathrm{Y})$ is the density of $X$ in aneighborhood of word $Y$. Here the neighborhood is defined as 50 words before or after any appearance of word $Y$. (There is a variety of neighborhood defunitions such as "100 surrounding words" (Yarowsky 1992) and "within a distance of no more than 3 words ignoring function words" (Digan et al. 1993).)

'The logarithm with 'p' is defined to be () for an argrmment less than 1. Negutive estimales were neglected because they ate mostly accidental except when $X$ and $Y$ are frequent enough (Church and Hanks 1989).

$A$ co-occurence vector of a word is defined as the list of co-oceurrence likelihood of the word with a certain set of origin words. We used the same set of origin words as for the distance vectors.

$C V[w]=\left(\begin{array}{c}I\left(w, O_{1}\right) \\ I\left(w, O_{2}\right) \\ \vdots \\ \vdots \\ I\left(w, O_{11}\right)\end{array}\right)$

Co-occurrence Vector.

When the frequency of $\mathrm{X}$ or $\mathrm{Y}$ is zero, we can not measure their co-occurence likelihood, and such cases are not exeeptional. This sparseness problem is well known and serious in the co-occurence statistics. We used as a corpus the 1987 Wall street Journal in the (C)-ROM 1 (199I), which hals a total of $20 \mathrm{M}$ words. The number of words which appeared at least once was about $50 \%$ of the total $62 \mathrm{~K}$ head words of CEl), and the percentage of the uord-origin pairs which appeared at least once was about $16 \%$ of total $62 \mathrm{~K} \times$ $1 \mathrm{~K}(=62 \mathrm{M})$ pairs. When the co-occurrence likelihood cau not be measured, the value $I(X, Y)$ was set to 0 . 


\section{Experimental Results}

We compared the two vector representations by using them for the following two semantic tasks. The first is word sense disambiguation (WSD) based on the similarity of context vectors; the second is the learning of positive or negative meanings from example words.

With WSD, the precision by using co-occurrence vectors from a $20 \mathrm{M}$ words corpus was higher than by using distance vectors from the CED.

\subsection{Word Sense Disambiguation}

Word sense disambiguation is a serious semantic problem. A variety of approaches lave been proposed for solving it. For example, Véronis and Ide (1990) used reference networks as neural networks, Mearst. (1991) used (shallow) syntactic similarity between contexts, Cowie et al. (1992) used simulated annealing for quick parallel disambiguation, and Yarowsky (1992) used co-occurrence statistics between words and thesaurus categories.

Our disambiguation method is based on the similarity of context vectors, which was originated by Wilks et al. (1990). In this method, a context vector is the sum of its constituent word vectors (except the target word itself). That is, the context vector for context,

$$
C: \ldots w_{-N} \ldots w_{-1} w w_{1} \ldots w_{N^{\prime}} \ldots,
$$

is

$$
V(C)=\sum_{i=-N}^{N^{\prime}} V\left(w_{i}\right)
$$

The similarity of contexts is measured by the angle of their vectors (or actually the inner product of their normalized vectors).

$$
\operatorname{sim}\left(C_{1}, C_{2}\right)=\frac{V\left(C_{1}\right)}{\left|V\left(C_{1}\right)\right|} \cdot \frac{V\left(C_{2}\right)}{\left|V\left(C_{2}\right)\right|}
$$

Let word $w$ have senses $s_{1}, s_{2}, \ldots, s_{m}$, and each sense have the following context examples.

\begin{tabular}{cc} 
Sense & Context Examples \\
\hline$s_{1}$ & $\mathrm{C}_{11}, \mathrm{C}_{12}, \ldots \mathrm{C}_{1 \mathrm{n}_{1}}$ \\
$\mathrm{~s}_{2}$ & $\mathrm{C}_{21}, \mathrm{C}_{22}, \ldots \mathrm{C}_{2 \mathrm{n}_{2}}$ \\
$\vdots$ & $\vdots$ \\
$\mathrm{s}_{\mathrm{m}}$ & $\mathrm{C}_{\mathrm{m} 1}, \mathrm{C}_{\mathrm{m} 2}, \ldots \mathrm{C}_{\mathrm{m} \mathrm{n}_{\mathrm{m}}}$
\end{tabular}

We infer that the sense of word $w$ in an arbitrary context $\mathrm{C}$ is $\mathrm{s}_{\mathrm{i}}$ if for some $\mathrm{j}$ the similarity, $\operatorname{sim}\left(\mathrm{C}, \mathrm{C}_{\mathrm{ij}}\right)$, is maximum among all the context examples.

Another possible way to infer the sense is to choose sense $s_{i}$ such that the average of $\operatorname{sim}\left(C, C_{i j}\right)$ over $\mathrm{j}=1,2, \ldots, \mathrm{n}_{\mathrm{i}}$ is maximum. We selected the first method because a peculiarly similar example is more important than the average similarity.

Figure 3 (next page) shows the disambiguation precision for 9 words. For each word, we selected two senses shown over each graph. These senses were chosen because they are clearly different and we could collect sufficient number (more than 20) of context examples. The names of senses were chosen from the category names in Roget's International 'Thesaurus, except organ's.

The results using distance vectors are shown by dots (••), and using co-occurrence vectors from the 1987 WSJ (20M words) by circles (o o o).

$A$ conlext size (x-axis) of, for example, 10 means 10 words before the target word and 10 words after the target word. We used 20 examples per sense; they were taken from the 1988 WSJ. The test contexts were from the 1987 WSJ: The number of test contexts varies from word to word (100 to 1000). The precision is the simple average of the respective precisions for the two senses.

The results of Fig. 3 show that the precision by using co-occurrence vectors are higher than that by using distance vectors except two cases, interest and customs. And we have not yet found a case where the distance vectors give higher precision. Therefore we conclude that co-occurrence vectors are advantageous over distance vectors to WSD based on the context similarity.

The sparseness problem for co-occurrence vectors is not serious in this case because each context consists of plural words.

\subsection{Learning of positive-or-negative}

Another experiment using the same two vector representations was done to measure the learning of positive or negative meanings. Figure 4 shows the changes in the precision (the percentage of agreement with the iuthors' combined judgement). The x-axis indicates the number of example words for each positive or negative pair. Judgement was again done by using the nearest example. The example and test words are shown in 'lables 1 and 2, respectively.

In this case, the distance vectors were advantageous. 'T'lue precision by using distance vectors increased to about $80 \%$ and then leveled off, while the precision by using co-occurrence vectors stayed around $60 \%$. We can therefore conclude that the property of positive-or-negative is reflected in distance vectors more strongly than in co-occurrence vectors. The sparseness problem is supposed to be a major factor in this case. 


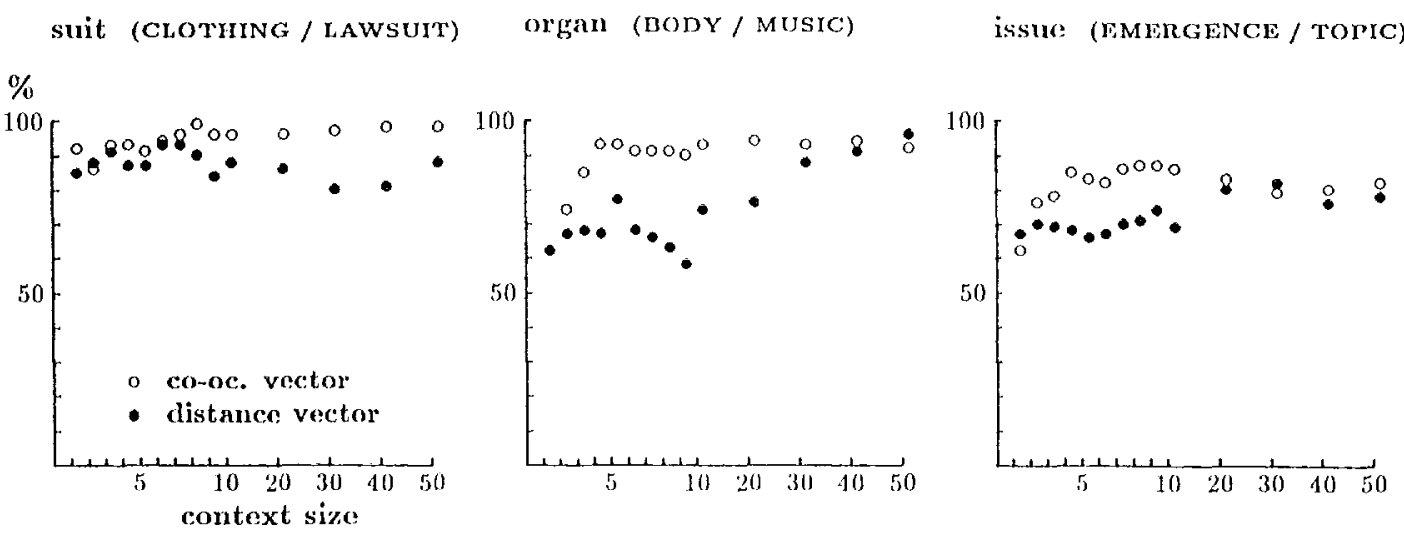

tank (CONTAINER / VEHICLE) order (COMMAND/ DEMAND) addross (Habitat / SPEECH)
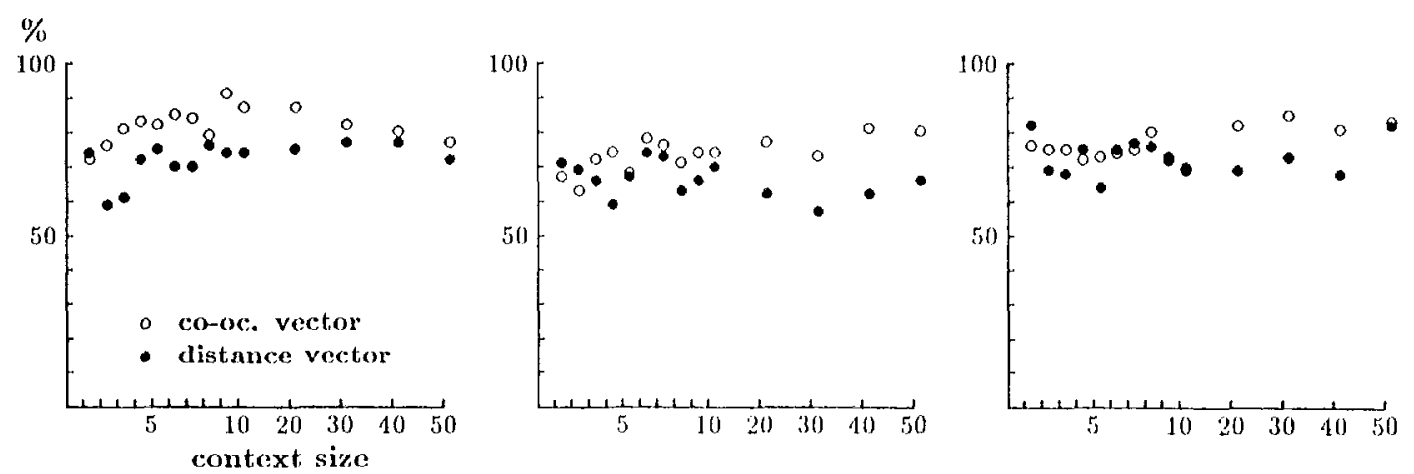

ruce (CLASS / OPPOSYTYON)

customs (IAIIT(1,.) / SEIVICE) interest (CURIOSITY / DENT)
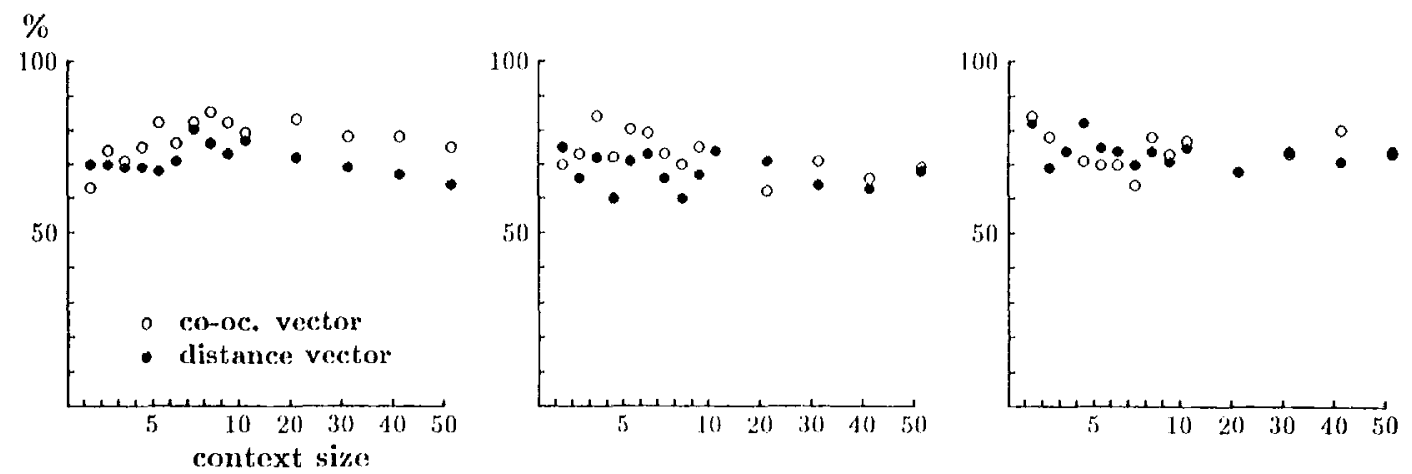

Fig. 3 Disambiguation of 9 words by using co-occurrence vectors(ooo) and by

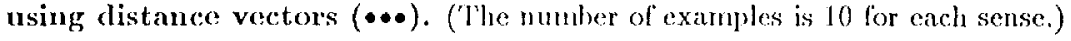




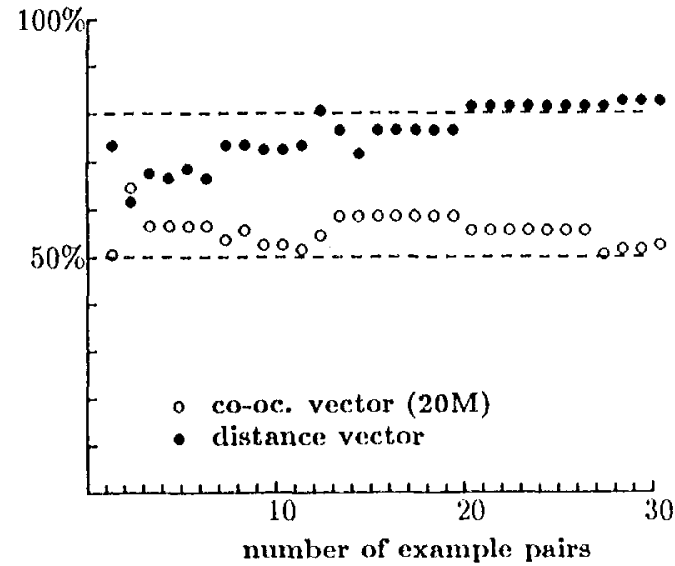

Fig. 4 Learning of positive-or-negative.

Table 1 Example pairs.

\begin{tabular}{rlllll}
\multicolumn{1}{l}{ positive } & negative & posilive & negative \\
1 & true & false & 16 & properly & crime \\
2 & new & wrong & 17 & succed & die \\
3 & better & disease & 18 & worth & violent \\
4 & clear & angry & 19 & friendly & hurt \\
5 & pleasure & noise & 20 & useful & punishunent \\
6 & correct & prin & 21 & success & poor \\
7 & pleasant & lose & 22 & interesting & badly \\
8 & suitable & destroy & 23 & active & fail \\
9 & clean & dangerous 24 & polite & suffering \\
10 & advantage & harm & 25 & win & enemy \\
11 & love & kill & 26 & improve & rude \\
12 & best & fear & 27 & favour & danger \\
13 & successful & war & 28 & development anger \\
14 & attractive & ill & 29 & happy & waste \\
15 & powerful & foolish & 30 & praise & doubt
\end{tabular}

Tuble 2 T'est words.

\begin{abstract}
positive (20 words)
\end{abstract}
balanced elaborate elation eligible enjoy fluent honorary honourable hopeful hopefully influential interested legible lustre normal recreation replete resilient restorative sincere

negative (30 words)

$\begin{array}{lllll}\text { confusion } & \text { cuckold } & \text { dally } & \text { dammation dull } \\ \text { ferocions } & \text { flaw } & \text { hesitate } & \text { hostage } & \text { huddle } \\ \text { inattentive } & \text { liverish } & \text { lowly } & \text { mock } & \text { neglect } \\ \text { queer } & \text { rape } & \text { ridiculous savage } & \text { scanly } \\ \text { sceptical } & \text { schizophrenia } & \text { scolr } & \text { scruffy } & \text { shipwreck } \\ \text { superstition sycophant } & \text { trouble } & \text { wicked } & \text { worliless }\end{array}$

\subsection{Supplementary Data}

In the experiments discussed above, the corpus size for co-occurrence vectors was set to $20 \mathrm{M}$ words ('87 WSJ) and the vector dimension for both co-occurrence and distance vectors was set to 1000 . IYere we show some supplementary data that support these parameter settings.

a. Corpus size (for co-occurrence vectors)

Figure 5 shows the change in disambiguation pre- cision as the corpus size for co-occurrence statistics increases from 200 words to $20 \mathrm{M}$ words. (The words are suit, issue and race, the context size is 10 , and the number of examples per sense is 10.) These three graphs level off after around $1 \mathrm{M}$ words. Therefore, a corpus size of $20 \mathrm{M}$ words is not too small.

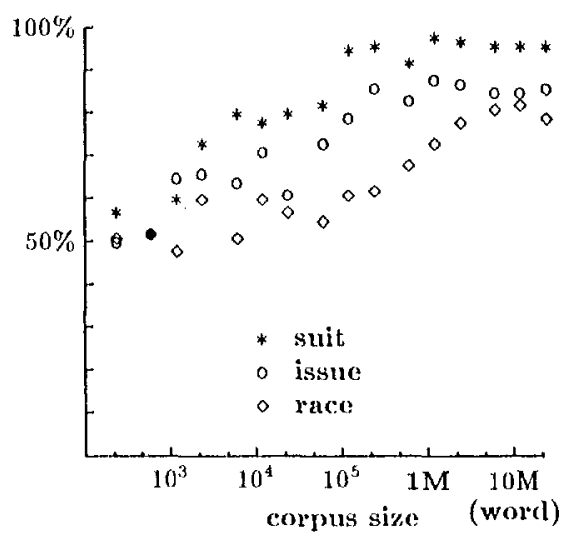

Fig. 5 Dependence of the disambiguation precision on the corpus size for co-occurrence vectors. context size: 10 , number of examples: 10/sense, vector dimension: 1000 .

\section{b. Vector Dimension}

Figure 6 (next page) shows the dependence of disanbiguation precision on the vector dimension for (i) co-occurrence and (ii) distance vectors. As for cooccurrence vectors, the precision levels off near a dimension of 100 . Therefore, a dimension size of 1000 is sufficient or even redundant. However, in the distance vector's case, it is not clear whether the precision is leveling or still increasing around 1000 dimension.

\section{Conclusion}

- A comparison was made of co-occurrence vectors from large text corpora and of distance vectors from dictionary definitions.

- For the word sense disambiguation based on the context similarity, co-occurrence vectors from the 1987 Wall Street Journal (20M total words) was advantageous over distance vectors from the Collins English Dictionary (60K head words + $1.6 \mathrm{M}$ definition words).

- For learning positive or negative meanings from example words, distance vectors gave remarkably higher precision than co-occurrence vectors. This suggests, though further investigation is recuired, that distance vectors contain some different semantic information from co-occurrence vectors. 
(i) by co-oc. vectors

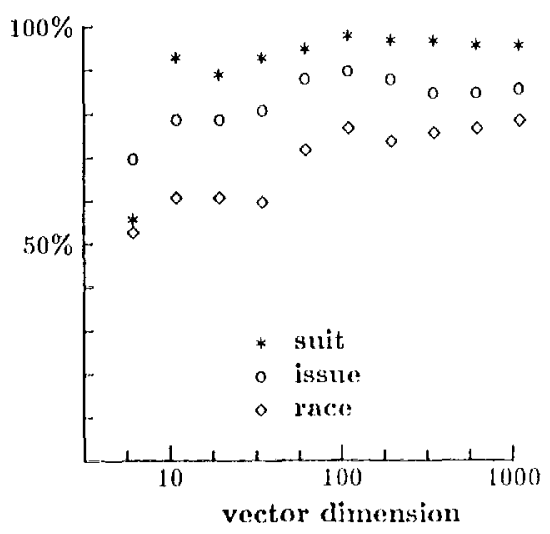

(ii) by distance vectors

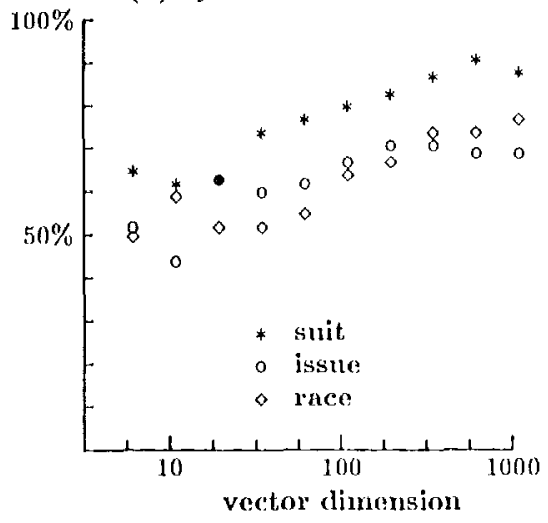

Fig. 6 Dependence on vector dimension for (i) cooccurrence vectors and (ii) distance vectors. context size: 10 , examples: 10/sense, corpus size for co-oc. vectors: $20 \mathrm{M}$ word.

\section{References}

Kenneth W. Church and Patrick IIanks. 1989. Word association norms, mutual information, and lexi-

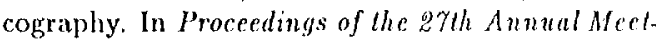
ing of the Association for Compulational Linguistics, pages 76-83, Vancouver, Camadia.

Jim Cowie, Joe Guthrie, and Louise Guthrie. 1992. Lexical disambiguation using simulated annealing. In Proceedings of COLING-92, pages 359-365, Nantes, France.

Iclo Dagan, Shaul Marcus, and Shaul Markovitch. 1993. Contextual word similarity and estimation from sparse data. In l'rocecdings of the $31 \mathrm{st} A n$ nual Meeting of the Associalion for Compulational Linguistics, pages 164-171, Columbus, Olio.

James Deese. 1962. On the structure of associative meaning. Psychological Review, 69(3):161-175).

Marti A. Hearst. 1991. Noun homograph disambiguation using local context in large text corpora. In
Procedings of the 7th Annual Conference of the University of Waterloo Center for the New OED and I'ext Research, pages 1-22, Oxford.

llideki Kozima and 'leiji Furugori. 1993. Similarity between words computed by spreading activation on an english dictionary. In Proceedings of $F A C L_{-}$ 93, pages 232-239, Utrecht, the Netherlands.

Mark Yiberman, editor. 1991. CD.ROM r. Association for Computational linguistics Data Collection Initiative, University of Pennsylvania.

Yoshihiko Nitta. 1988. 'The referential structure of the word definitions in ordinary dictionaries. In Prococdings of the Workshop on the Aspects of Lexicon for Nalural Language Processing, LNL88-8, JSSST, pages 1-21, Hukuoka University, Japan. (in Japanese).

Yoshihiko Nitta. 1993. Referential structure - a mechanism for giving word-definition in ordinary lexicons. In C. Lee and B. Kang, editors, Language, Information and Computation, pages 99110. Thadhalssa, Seoul.

Yoshiki Niwa and Yoshiliko Nitta. 1993. Distance vector repressentation of words, derived from reference networks in ordinary dictionaries. MCCS 93253, Compuling Research Laboratory, New Mexico State University, Las Cruces.

C. li. Osgood, G. F' Such, and P. II. 'Tamnenbaum. 1957. The Measurement of Meaning. University of lllinois Press, Urbana.

Fernando Pereira, Naftali Tishby, and Lillian Lee. 1993. Djstributional clustering of english words. In Procecdings of the $31 \mathrm{st}$ Annual Mecting of the Association for Computational Linguistics, pages 183-190, Columbus, Ohio.

Paul Procter, editor. 1978. Longman Dictionary of Contemporary linglish (LDOCW). Longman, IIarlow, Fssex, first edition.

Irimrich Schütze. 1993. Word space. In J. D. Cowan S. J. Ilanson and C. T. Ciles, elitors, Advances in Neural Information Processing Systems, pages 895 902. Morgan Kanfmamn, Sau Mateo, Californit.

John Sinclair, editor. 1987. Collins COBUThD Finglish Language Dictionary. Collins and the University of Birmingham, I,ondon.

Jean Véronis and Nancy M. Idle. 1990. Word sense disambiguation with very large neural networks extracted from machine readable dictionaries. In Procedings of COLING-90, pages 389-394, Jelsinki.

Yorick Wills, Dan Fass, Cheng ming Gro, Jarnes li. MeDonald, 'Tony Plate, and Brian M. Slator. 1990. Providing machine tractable dictionary tools. Machine 'Transtation, 5(2):99-154.

David Yarowsky, 1992. Word-sense disambiguation using statistical models of roget's categories trained on large corpora. In procedings of COLING-92, pages 454-460, Nantes, lrance. 